\title{
Capacity and Energy Local Flexibility Markets for Imbalance and Congestion Management
}

\author{
Ángel Paredes, José A. Aguado \\ angelparedes@uma.es, jaguado@uma.es \\ Department of Electrical Engineering \\ Industrial Engineering School - University of Málaga \\ C/ Dr. Ortiz Ramos s/n. Campus de Teatinos. 29071 Málaga., Spain
}

\begin{abstract}
Local Flexibility Markets (LFMs) are a type of marketplace where flexibility products are traded to solve network issues. In the last several years, efforts were made in the field of LFM for imbalance and congestion management, but they only trade with energy products with highly complex mathematical descriptions. This paper presents a description of an efficient LFM formulation where both capacity and energy products are considered through a linear programming (LP) optimization problem to clear the LFM in a centralized manner. It is assumed that flexibility assets present in the distribution network are managed by Aggregators (Aggs). Results from this centralized approach are provided under three different scenarios, demonstrating the feasibility of the proposed approach to solve network issues at a distribution level.
\end{abstract}

Keywords—Local Flexibility Market, Energy Products, Capacity Products, Congestion, Imbalance.

\section{NOMENCLATURE}

Main sets, indexes, parameters, and variables are listed in this section, other derivatives are introduced in the text.

$l, f, g, s, \quad$ Sets of static loads, flexible loads, generation assets, storage systems, time, slack node,

$t, s l k, i \in \mathbb{N} \quad$ and grid nodes.

$x \in\{f, g, s\} \quad$ Set of flexible assets connected to the grid.

$j \in i \quad$ Set of nodes connected between them.

$B_{i j} \quad$ Susceptance of line $i j(\mathrm{~S})$.

$\theta_{i j} \quad$ Difference of voltage angle between node $i$ and $j(\mathrm{rad})$.

$\omega \quad$ Product quantity ( $\mathrm{kW}$ or $\mathrm{kWh})$.

$S \quad$ Product price $(€ / \mathrm{kW}$ or $€ / \mathrm{kWh})$.

$C_{x, t} \quad$ Cost function for asset $x$ in interval $t(€)$.

soc $_{s} \quad$ State of Charge of storage system $s(\mathrm{kWh})$.

$\eta_{s}^{C}, \eta_{s}^{D} \quad$ Charging and discharging efficiencies of the battery $s$ (p.u.).

$p \quad$ Active power injection $(\mathrm{kW})$.

Notation: variables are small letters, e.g., $\omega$. Upper and lower limits of $\omega$ are noted by $\bar{\omega}$ and $\underline{\omega}$, respectively. Parameters are noted by capital letters, e.g., $B_{i j}$. Energy and capacity products are noted by superscript $e$ and $c$, respectively, and direction is noted by superscript $u$ for upward and $d$ for downward directions, e.g., $\omega_{x, t}^{c, d}$ or $\omega_{x, t}^{e, u}$. Scheduled power injection and aftermarket injection are noted by $P R$ and $A M$, respectively, e.g., $P_{l, t}^{P R}$ or $p_{f, t}^{A M}$.

\section{ABBREVIATIONS}

Abbreviations used in the paper are listed in this section:

$\begin{array}{cll}\text { RE } & \text { Renewable Energies } & \text { DSR } \\ \text { PV } & \text { Photovoltaics systems } & \text { NLP } \\ \text { DER } & \text { Distributed Energy Resource } & \text { LP } \\ \text { LFM } & \text { Local Flexibility Market } & \text { FL } \\ \text { DSO } & \text { Distribution System Operator } & \text { FG } \\ \text { BRP } & \text { Balance Responsible Party } & \text { SL } \\ \text { Agg } & \text { Aggregator } & \text { BESS } \\ \text { LMO } & \text { Local Market Operator } & \text { SOC }\end{array}$

Demand Side Response

Non-linear programming

Linear programming

Flexible Load

Flexible Generator

Static Load

Battery Energy Storage System

State of Charge 


\section{INTRODUCTION}

Deployment of RE has significantly increased over last years. Installed renewable capacity has increased an $89 \%$ in the last 10 years in Europe [1]. For instance, Spain has achieved a 50\% of RE production share in 2020 [2]. Uncontrollability of PV and wind energy is one of the main shortcomings when integrating RE. Part of this deployment is done at distribution level with DERs, usually, PV systems. Consequently, distribution grids face challenges such as imbalances, voltage deviations and line congestions.

In this context, LFMs present a solution to the intermittent behaviour of DERs, using flexibility products to solve those issues. However, currently LFMs suffer from a lack of liquidity [3], what motivates a study about the influence of a Local Capacity Market working together with a Local Energy Market.

In the literature, there exist a wide type of methods to clear local marketplaces. Focusing on market-based methods, those techniques includes: LFMs, Price-based control techniques and Transactive Energy approaches [4]. Market schemes are preferred over price based control techniques as its protect to endcustomers, being also aligned with EU regulations [5]. Besides, offering high quality solutions with local resources, local communities running LFMs are emerging at organizational level [6].

For the purpose of this paper, LFM is defined as a type of marketplace where it is possible to trade electricity products in geographically limited areas, that is, small towns, neighbourhoods, communities or districts [7]. Therefore, they involve several agents who sell and buy electricity products, a market operator, and a clearing mechanism that match offers with asks [8].

The number of agents included in the market greatly varies depending on the LFM design. However, main actors are: DSO, BRPs, Agg and, LMO [9]. Those stakeholders are interested in participating in the market as DSOs solve congestion and voltage deviations, BRPs optimize their portfolios, and Aggs obtain benefits managing their assets.

Nevertheless, the role of each stakeholder differs among proposals. Reference [10], considers Agg as the LMO, providing different services to DSO and BRPs. Market defined in [11], conversely, does not include BRP figure, enhancing Aggs as the one who oversees the market, collecting bids and asks. Alternatively, reference [12] proposes a market structure where no Agg is presented, and flexibility is traded considering only end users. Moreover, authors of reference [13] acknowledge energy communities as a key player when defining market structure. Regardless of the approach considered, none of them clearly explain the relation between the prices of flexibility products and the wholesale market.

From an operational viewpoint, several LFM pilot projects are being developed over the European Union. Some examples are: IREMEL [14], InterFlex [15], EMPOWER [10] and DREAM-GO [13]. The Spanish project IREMEL addresses three main mechanisms for DSO to obtain flexibility: Congestion Management, Local Flexibility Products and long-term contracts using Aggs. The main objective of InterFlex project is to create a local energy market considering Aggs, Industrial Customers and Local Energy Communities. It focuses on both centralized and decentralized versions (P2P) of the market. EMPOWER and DREAM-GO situate Aggs as the central actor of the LFM providing DSR services to DSO.

Social welfare [16] or cost minimization for flexibility procurement are the usual objectives seek while defining the LFM. Costs optimization, depending on the viewpoint considered, can be minimized for Agg [10], LMO [11] and, DSO [17]. All references work with complex mathematical problems using Mixed Integer Linear Programming or NLP techniques. Despite the fact that LP methodologies has been proposed in the field of bi-level optimization [18], little contributions are made when considering LFM centralized approach.

In terms of flexibility products, reference [9] identifies three main energy products to be traded in distribution systems: DSR from FLs, generation curtailment from FGs and storage from BESSs. It also highlights the Agg figure as the manager of modest prosumers. Nevertheless, although being object of deep analysis, capacity products have not been addressed with enough detail in the literature under a LFM context. 


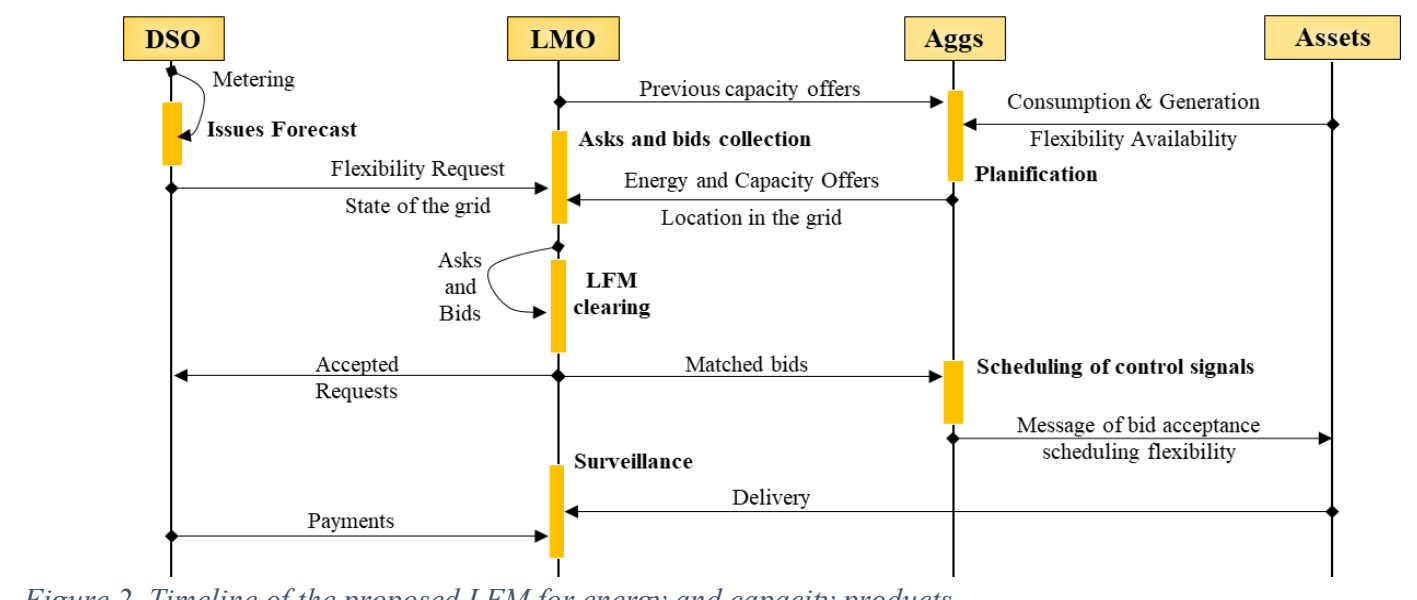

Figure 2. Timeline of the proposed LFM for energy and capacity products.

In this paper, a LFM is presented from the LMO viewpoint. It considers capacity and energy products from FLs, FGs and BESSs. Market formulation addresses cost minimization considering a linearized approach of the NLP constraints. Hence, an approximation to the global optimal solution is ensured. Main contributions of this paper are:

1. Clear and concise energy and capacity products definitions in a LFM context.

2. $\quad$ Linearized approach for non-linear restrictions of the centralized LFM problem from LMO viewpoint.

3. A methodology that includes Energy and Capacity products in one single LFM, increasing market liquidity.

The remaining of the paper is organized as follows. Section II introduces the LFM formulation, define energy and capacity products explaining the linearization process. Section III shows a case of study where the proposed market behaviour is investigated. Lastly, Section IV concludes the paper.

\section{Methodology and Problem Formulation}

In this section, the proposed market is described and formulated. The LFM presented is based on reference [7] considering, also, the Agg figure as the key enabler for prosumers trading flexibility. A deterministic framework of the problem is presented. Since market is cleared each 15 min during all day, uncertainties are considered negligible.

For the sake of clarity, Figure 1 shows a general view of the market structure and participating agents. Besides of that, Figure 2 presents the timeline sequence among market participants. As shown, main steps involve; firstly, DSO and Aggs forecasting and planning their energy asks and bids for the current period and the capacity asks and bids for the next period; secondly, LMO, clearing the market and, sending results to agents; lastly, Aggs sending control signals to assets while LMO looking the market out. In each market clearing, energy products are matched for time slot $t$ and capacity products are cleared for time slot $t+1$. Pre-allocation of the capacity products ensures liquidity of energy products in the following period as Figure 3 explains.

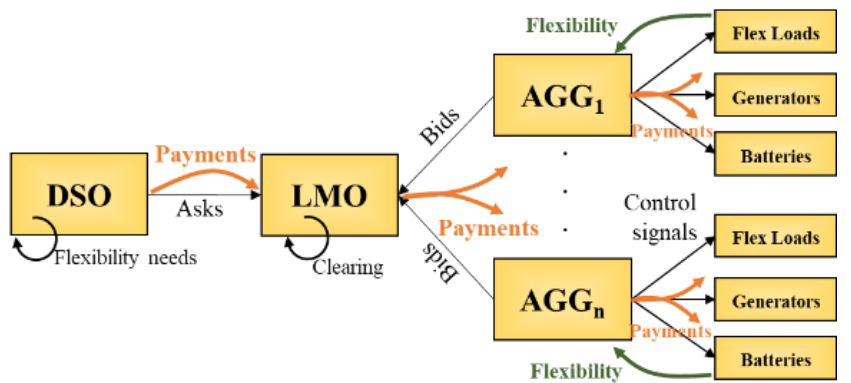

Figure 1. Market structure of asks, bids, control signals and payments of the centralized local flexibility market.

Agents presented in the market are aligned with the EU pilot projects mentioned, especially with EMPOWER and DREAM-GO, due to the Aggs' role. Flexibility is obtained from prosumers organized in three main categories: FLs, FGs and BESSs. Those agents offer different energy and capacity products as further sections explain. 


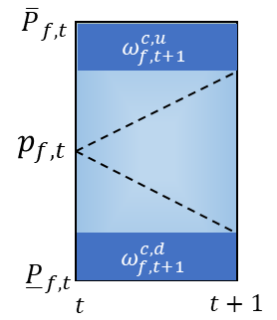

(a) FLs

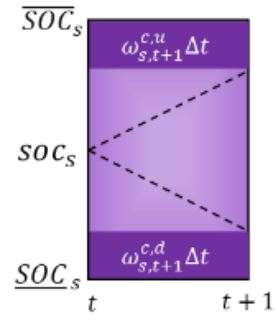

(b) $B E S S S$

Figure 3. Ensuring availability of energy flexibility products in $t+1$ using capacity products.

Capacity products are cleared in advance and set an upper bound for the quantity of energy product traded in the market. With this use of products, DSO incentivize prosumers, ensuring availability of flexibility in prevision of future grid issues. Since LFM is presented from a LMO perspective, the objective function ensures cost minimization of overall flexibility acquisition, leading to market efficiency where only the minimum possible quantity of products is traded to solve DSO contingency.

The following section defines flexibility products, formulates restrictions of the LP-LFM and carry the proposed linearization out.

\section{A. Flexibility Products definition.}

In this section, flexibility products are defined. Before the LFM, agents have bought and sold energy at the marginal price $S_{t}^{S}$ in the wholesale market. In this situation, they have no incentive to participate in a LFM if no benefit is present. However, DSO can offer a trade-off, incentivizing trading among prosumers. Thus, loads will reduce its consumption if they receive an offer of $S_{t}^{e, d}>S_{t}^{s}$ for their products and will increase it if the ask involves energy at a price $S_{t}^{e, u}<S_{t}^{s}$. Same reasoning can be done for BESSs. Nevertheless, for FGs, offering only curtailment of its generation, as their downward direction is equivalent to an increase in the consumption, they bid at a price $S_{g, t}^{e, d}<S_{t}^{s}$.

FLs are assumed to freely modify their consumption in a limited range in both directions. Cost for procuring those products is detailed in equations (1) and (2) for each FL. These equations consider the wholesale energy price showing costs incurred by DSO when purchasing flexibility products.

$$
\begin{gathered}
C_{f, t}^{e}=\left(S_{t}^{s}-S_{f, t}^{e, u}\right) \cdot \omega_{f, t}^{e, u}+\left(S_{f, t}^{e, d}-S_{t}^{s}\right) \cdot \omega_{f, t}^{e, d} \forall t, \forall f \\
C_{f, t}^{c}=S_{f, t}^{c, u} \cdot \omega_{f, t}^{c, u}+S_{f, t}^{c, d} \cdot \omega_{f, t}^{c, d} \forall t, \forall f
\end{gathered}
$$

Energy and capacity products are related as no asset can trade energy products far away than previously set capacity product. Then, $\omega_{f, t}^{e, u} \leq \omega_{f, t}^{c, u} \cdot \Delta t$ and $\omega_{f, t}^{e, d} \leq \omega_{f, t}^{c, d} \cdot \Delta t$ for all $t>t_{1}$. It is important, also, to clarify that simultaneous trade of upward and downward products are not allowed. For that reason, non-linear restriction (3) is stablished. Moreover, since energy products are dependent of capacity, no simultaneity is also achieved. Proposed restriction ensures that only one of the directions is activated, as the other necessarily needs to be zero to satisfy equation (3).

$$
\omega_{f, t}^{c, u} \cdot \omega_{f, t}^{c, d}=0 \quad \forall t, \forall f
$$

Increase of liquidity using capacity products is achieved, limiting energy products bounds in time $t$ in prediction of what will happen in period $t+1$ [19]. As Figure 3 (a) shows, consumption of FL $f$ is limited in period $t$ using capacity products. Then $\underline{P}_{f, t}+\omega_{f, t+1}^{c, d} \leq p_{f, t} \leq \bar{P}_{f, t}-\omega_{f, t+1}^{c, u}$, what ensures availability of energy products in $t+1$.

For FGs, PV is the main option when considering DERs in distribution networks. As a result, FGs are assumed to only provide flexibility in downward direction. They can only curtail their production; in any case can they increase it. Costs for flexibility procurement of energy and capacity products are shown in equations (4) and (5).

$$
\begin{gathered}
C_{g, t}^{e}=\left(S_{t}^{S}-S_{g, t}^{e, d}\right) \cdot \omega_{g, t}^{e, d} \quad \forall t, \forall g \\
C_{g, t}^{c}=S_{g, t}^{c, d} \cdot \omega_{g, t}^{c, d} \quad \forall t, \forall g
\end{gathered}
$$

Also, FGs capacity and energy products are related considering $\omega_{g, t}^{e, d} \leq \omega_{g, t}^{c, d} \cdot \Delta t$ for all FGs.

BESSs are key elements for procuring flexibility as are the only participants with the ability of storage energy. Costs of those products are detailed in equations (6) and (7).

$$
\begin{gathered}
C_{s, t}^{e}=\left(S_{t}^{s}-S_{s, t}^{e, u}\right) \cdot \omega_{s, t}^{e, u}+\left(S_{s, t}^{e, d}-S_{t}^{s}\right) \cdot \omega_{s, t}^{e, d} \forall t, \forall s \\
C_{s, t}^{c}=S_{s, t}^{c, u} \cdot \omega_{s, t}^{c, u}+S_{s, t}^{c, d} \cdot \omega_{s, t}^{c, d} \forall t, \forall s
\end{gathered}
$$


Consistently with previous presented products, BESSs energy and capacity products are related between each other considering $\omega_{s, t}^{e, u} \leq \omega_{s, t}^{c, u} \cdot \Delta t$ and $\omega_{s, t}^{e, d} \leq \omega_{s, t}^{c, d} \cdot \Delta t$ for all BESSs. Moreover, as a matter of fact, BESSs do only store energy, in consequence, their net balance is zero if a sufficient long period is considered. Thus, SOC is assumed to have the same value in the first and last period, $s o c_{s, t_{1}}=s o c_{s,|T|}$. In the case of BESSs, simultaneous activation of upward and downward directions is not allowed defining restriction (8).

$$
\omega_{s, t}^{c, u} \cdot \omega_{s, t}^{c, d}=0, \quad \forall t, \forall s
$$

Previous reasoning explained for FLs, can be also applied to BESSs. Considering a need of energy in $t+1$ of $\omega_{s, t+1}^{e, u}$, in time $t+1$, BESSs should have a SOC lower than $\overline{S O C}_{s}-\omega_{s, t+1}^{c, u} \Delta t$. If the need is in the downward direction, minimum level of SOC will be $S O C_{S}+\omega_{s, t+1}^{c, d} \Delta t$. Then, pre-allocating capacity products will ensure availability of energy products, increasing liquidity of LFM as explained in Figure 3 (b).

Aggs manage assets in a specific area, collecting their flexibility and bidding in the LFM. Costs for energy and capacity products for an Agg is described in equations (9) and (10), respectively.

$$
\begin{aligned}
& C_{a, t}^{e}=\sum_{f \in a} C_{f, t}^{e}+\sum_{g \in a} C_{g, t}^{e}+\sum_{s \in a} C_{s, t}^{e} \quad \forall t, \forall a \\
& C_{a, t}^{c}=\sum_{f \in a} C_{f, t}^{c}+\sum_{g \in a} C_{g, t}^{c}+\sum_{s \in a} C_{s, t}^{c} \quad \forall t, \forall a
\end{aligned}
$$

\section{B. Restrictions.}

Balance restriction is defined considering programmed wholesale market schedule and the injections after clearing LFM. Aftermarket power consumption is defined considering energy products traded as $p_{f, t}^{A M}=$ $P_{f, t}^{P R}+1 / \Delta \mathrm{t} \cdot\left(\omega_{f, t}^{e, u}-\omega_{f, t}^{e, d}\right)$ for all FLs, $p_{s, t}^{A M}=1 / \Delta \mathrm{t} \cdot\left(\omega_{s, t}^{e, u}-\omega_{s, t}^{e, d}\right)$ for all BESSs and $p_{g, t}^{A M}=P_{g, t}^{P R}-$ $1 / \Delta \mathrm{t} \cdot \omega_{g, t}^{e, d}$ for all FGs. Then, equation (11) states power equality after the market clearing, preventing deviations from the original schedule and ensuring solving issues locally. This restriction represent DSO ask for balance to the local flexibility market.

$$
\sum_{l} P_{l, t}^{P R}-\sum_{x} P_{x, t}^{P R}=\sum_{l} P_{l, t}^{A M}-\sum_{x} p_{x, t}^{A M} \forall t
$$

On the other hand, BESSs charging and discharging efficiencies $\left(\eta_{s}^{C}, \eta_{s}^{D}\right)$ are also considered. For the sake of simplicity, they are set constant equals to $95 \%$. Then, SOC restrictions for energy market $\left(\operatorname{soc}_{s, t}\right)$ are considered.

$$
\begin{gathered}
\operatorname{soc}_{s, t}=\operatorname{soc}_{s, t-1}+\left(\eta_{s}^{C} \omega_{s, t}^{e, u}-\frac{1}{\eta_{s}^{D}} \omega_{s, t}^{e, d}\right) \quad \forall s \forall t \\
\underline{\operatorname{SOC}}_{s} \leq \operatorname{soc}_{s, t} \leq \overline{\operatorname{SOC}}_{s} \quad \forall s, \forall t
\end{gathered}
$$

Output power extracted from battery cannot be greater than the nominal power of the inverter $\left(P_{S}^{I N V}\right)$ connected to. Then, $\omega_{s, t}^{c, u} \leq P_{s}^{I N V}$ and $\omega_{s, t}^{c, d} \leq P_{s}^{I N V}$ for all BESSs.

Network restrictions should also be described. Grid will be modelled using a linear approach, considering that no voltage issues are present. Linear modelling considers all node voltages remain stable at voltage basis $\left(V_{i}=1\right)$, and that grid losses are negligible $\left(G_{i j} \approx 0\right)$. Then, network constraints for linear modelling of the distribution network are presented in equations (14) and (15)

$$
\begin{gathered}
\sum_{j \in i} B_{i j}\left(\theta_{i, t}-\theta_{j, t}\right)=p_{s l k, t}-P_{l, t}^{A M}+p_{x, t}^{A M} \forall i \forall t \\
-\bar{P}_{i j} \leq B_{i j}\left(\theta_{i, t}-\theta_{j, t}\right) \leq \bar{P}_{i j} \forall i, j \in i \forall t
\end{gathered}
$$

\section{Linearization of the problem.}

To effectively linearize NLP restrictions, firstly it is necessary to convert them into quadratic ones. To this aim, a variable change will be used. After that, $n$ tangent lines are used to linearize quadratic restrictions. Non-linear restriction is expressed in term of upward $\left(\omega_{x, t}^{c, u}\right)$ and downward $\left(\omega_{x, t}^{c, d}\right)$ directions for a given asset $x$. Firstly, variable change is satisfied considering $y_{x, t}^{c, u}=1 / 2 \cdot\left(\omega_{x, t}^{c, u}+\omega_{x, t}^{c, d}\right)$ and $y_{x, t}^{c, d}=1 / 2$. $\left(\omega_{x, t}^{c, u}-\omega_{x, t}^{c, d}\right)$, resulting equation (16).

$$
\left(y_{x, t}^{c, u}\right)^{2}-\left(y_{x, t}^{c, d}\right)^{2}=0 \quad \forall x, \forall t
$$

As can be noted, upward and downward variables are now separated, and restriction can be relaxed to a set of $n$ regions defined by $n$ tangent lines to the quadratic function, as Figure 4 represents. To this end, an auxiliar variable $z$ is defined for each $y$ variable. 


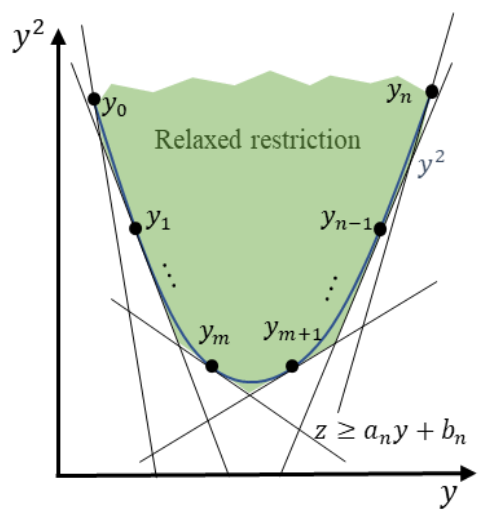

Figure 4. Relaxation of the convex quadratic restrictions obtained after a variable change over non-linear restriction.

Tangent lines are defined with $n$ linear coefficients $a_{n}$, as the slope, and $b_{n}$, as the independent term. Relaxed feasible region has the form defined in equation (17).

$$
z_{x, t} \geq a_{n} \cdot y_{x, t}+b_{n}
$$

$z_{x, t}$ variables must be minimized in the objective function also to ensure convergence on the lower boundary, approximating quadratic restriction and, consequently, satisfying non-linear restriction as Figure 4 shows.

\section{Objective function.}

The objective function includes two terms, the first one is the total cost of procuring flexibility from the aggregators to the DSO, and second ensures converge in the lower bound of the linearized restrictions as equation (18) estates. $C_{a, t}^{e}$ represents both flexibility direction of energy products, and $C_{a, t+1}^{c}$ also for capacity products.

$$
\min _{v a r s} \sum_{a} \sum_{t}\left(C_{a, t}^{e}+C_{a, t+1}^{c}\right)+\sum_{x} \sum_{t}\left(z_{x, t}^{c, u}+z_{x, t}^{c, d}\right)
$$

\section{CASE OF StUdy AND Simulation Results}

The proposed LFM formulation is tested on two different distribution networks based on the IEEE 34 radial distribution feeder. First topology, shown in Figure 5, is a piece of the second network, illustrated in Figure 6 which represents a region with different areas. SLs, FLs, FGs and BESSs are located in the scheme using coloured circles. Their profiles are synthetically generated considering ordinary profiles of residential users, product orientated industry and PV for a sunny day in March at Málaga (Spain). It is assumed that each radial feeder is managed by an Agg and only a DSO oversee the whole system. First 34-bus network has a static demand of $500 \mathrm{~kW}$, consisting in $15 \mathrm{SLs}, 1 \mathrm{FL}$ and 1 BESSs. The second 160-bus network has a static demand of 60,5 MW with 75 SLs, 20 FLs, 20 FGs, 20 BESSs and 5 Aggs. Each Agg will manage one feeder, e.g., Agg 1 will manage assets downstream node 000 and, Agg 2 of node 200, and so on.

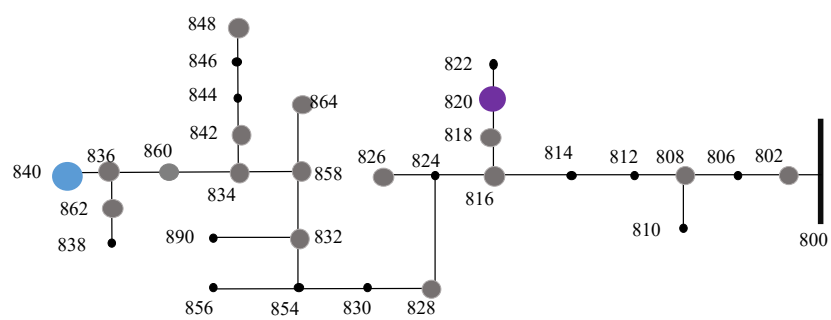

Figure 5. IEEE 34 radial distribution feeder. Gray circles represent SLs, blue FLs, purple BESSS. Slack node: 800.

Test scenarios aim to demonstrate the effectiveness of the proposed LFM for DSO imbalance and congestion management. For all test scenarios, wholesale prices are obtained from OMIE [20] for day March $22^{\text {nd }}, 2021$. Products offers are randomly generated in a range of $0,05 € / \mathrm{kWh}$ with respect to wholesale price. Three different scenarios are investigated, first one demonstrates liquidity increase, second one aims to solve an imbalance assuming the total disconnection of one of the SLs, and third one solves a congestion in a transmission line. First scenario is tested on the 34-bus network aiming to demonstrate the increase in liquidity. Then, second and third scenarios are tested on the 160-bus network to demonstrate imbalance and congestion solving effectiveness. 
The simulations are performed on a personal computer with a quad-core Intel i7 $2.60 \mathrm{GHz}$ with $16 \mathrm{~GB}$ of RAM. Generalized Algebraic Modelling System software (GAMS) is used and CPLEX solver is selected to solve LP-LFM. Computation times are plotted in Figure 10 and Figure 14 against the number of tangents lines. The total time spent in the finest scenario $(n=200)$ was $213.88 \mathrm{~s}$ for imbalance solving and $225.48 \mathrm{~s}$ for congestion solving.

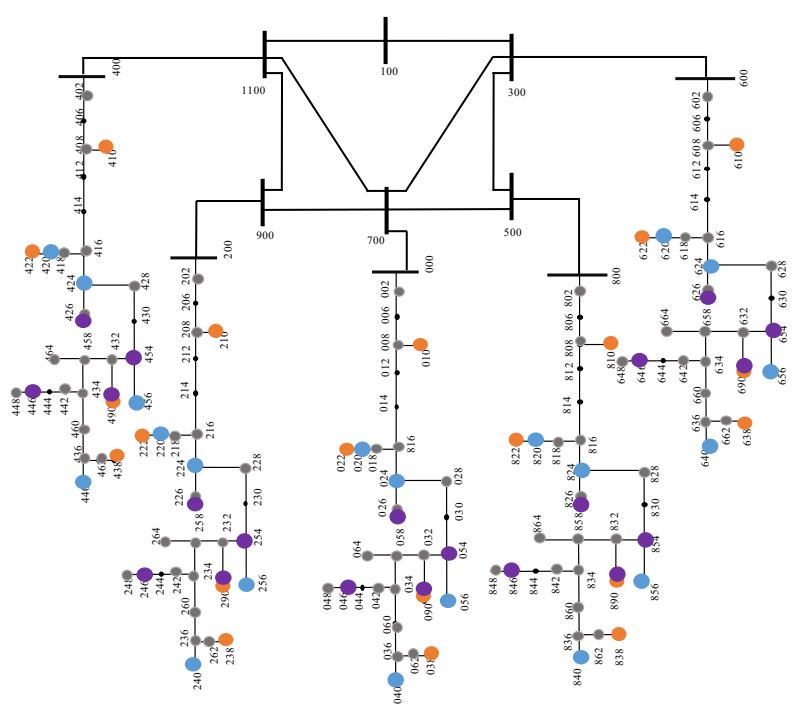

Figure 6. 160-bus network based on IEEE 34 radial distribution feeder. Gray circles represents SLs, orange FGs, blue FLs and purple BESSs. Slack node: 100.

\section{A. Liquidity improving}

First scenario considers a congestion on the line 858-834 limited to $300 \mathrm{~kW}$. Only two periods and a FL and a BESS are considered. BESS is fully charged at initial time $(300 \mathrm{kWh})$. Forecasted power flow predicts a congestion as table I shows. Under this situation, unless capacity products are considered, congestion cannot be avoided. Energy-LFM results infeasible in the second period as BESS is fully charged and cannot balance grid. However, as tables II and III show, Energy and Capacity LFM can anticipate this situation and pre-allocate the BESS capacity products discharging in the first period. Then, market liquidity is increased as battery can now operate in the second period solving the congestion while balancing the grid.

Table I. Scheduled, Energy LFM (E-LFM) and Energy and Capacity LFM (EC-LFM) power flow inline 858-834.

\begin{tabular}{|l|c|c|c|}
\hline & Scheduled & E-LFM & EC-LFM \\
\hline $\boldsymbol{t}_{\mathbf{1}}$ & 284.7 & 284.7 & 298.6 \\
\hline $\boldsymbol{t}_{\mathbf{2}}$ & 314.7 & 314.7 & 300.0 \\
\hline
\end{tabular}

Table II. Energy, capacity products and SOC for BESS after ECM clearing.

\begin{tabular}{|l|l|l|l|l|l|}
\hline & $\boldsymbol{\omega}_{\text {s.t }}^{\boldsymbol{e}, \boldsymbol{u}}$ & $\boldsymbol{\omega}_{\text {s.t }}^{\boldsymbol{c}, \boldsymbol{u}}$ & $\boldsymbol{\omega}_{\text {s.t }}^{\boldsymbol{e}, \boldsymbol{d}}$ & $\boldsymbol{\omega}_{\text {s.t }}^{\boldsymbol{c}, \boldsymbol{u}}$ & $\boldsymbol{S O C}$ \\
\hline $\boldsymbol{t}_{\mathbf{1}}$ & & & 3.49 & 13.95 & 296.33 \\
\hline $\boldsymbol{t}_{\mathbf{2}}$ & 3.67 & 14.69 & & & 299.82 \\
\hline
\end{tabular}

Table III. Energy and capacity products for FL after ECM clearing.

\begin{tabular}{|l|l|l|l|l|}
\hline & $\boldsymbol{\omega}_{\text {f.t }}^{\boldsymbol{e}, \boldsymbol{u}}$ & $\boldsymbol{\omega}_{\text {f.t }}^{\boldsymbol{c}, \boldsymbol{u}}$ & $\boldsymbol{\omega}_{\text {f.t }}^{\boldsymbol{e}, \boldsymbol{d}}$ & $\boldsymbol{\omega}_{\text {f.t }}^{\boldsymbol{c}, \boldsymbol{u}}$ \\
\hline $\boldsymbol{t}_{\mathbf{1}}$ & 3.49 & 13.95 & & \\
\hline $\boldsymbol{t}_{\mathbf{2}}$ & & & 3.67 & 14.69 \\
\hline
\end{tabular}

\section{B. Imbalance solving}

The second scenario considers the situation of an imbalance created by the disconnection of SL 75 (node 848). To demonstrate the effectiveness of the proposed LFM to solve imbalances, Figure 7 is presented. SL 75 has a near constant consumption profile of product-oriented industries. Disconnection is solved using all presented products.

Positive values of the figure axis represent upward direction of the products, and negatives, downward. As batteries are urged to maintain its SOC at the end of the day, they charge while FGs can cover their consumption and sell downward products rest of the time. 


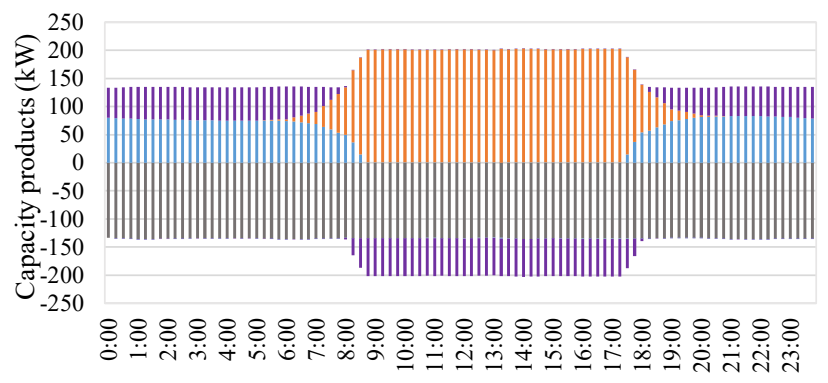

Figure 7. FLs (blue), FGs (orange), BESSs (purple) capacity products traded in the LFM to solve SL75 (grey) imbalance.

An hourly distribution of the costs is presented in Figure 8. Total costs amount to $166 €$ for a total of $4,491.2 \mathrm{kWh}$ of energy products traded and $17,964.8 \mathrm{~kW}$ of capacity products pre-allocated. In this scenario, Aggs share of products is presented in Figure 9. Agg 5 stand out with most of the trade of FGs downward flexibility products, rest of products are similarly shared among Aggs.

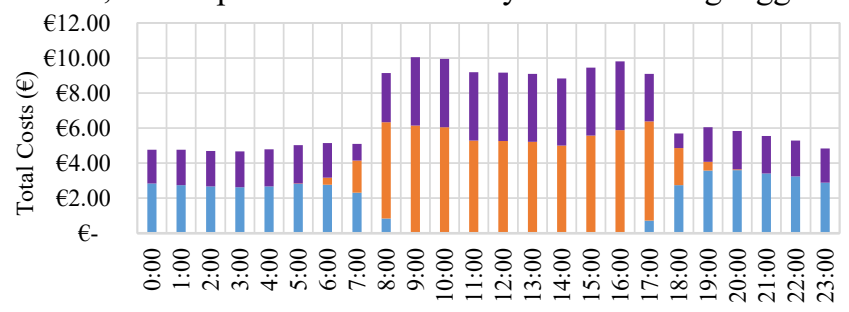

Figure 8. FLs (blue), FGs (orange), BESSs (purple) hourly cost for flexibility dispatch in the imbalance scenario. Total FLs costs: 42.66€; Total FGs costs: 60.33€; Total BESSs costs: 63.01€. Quantity traded: 4,491.2 kWh.

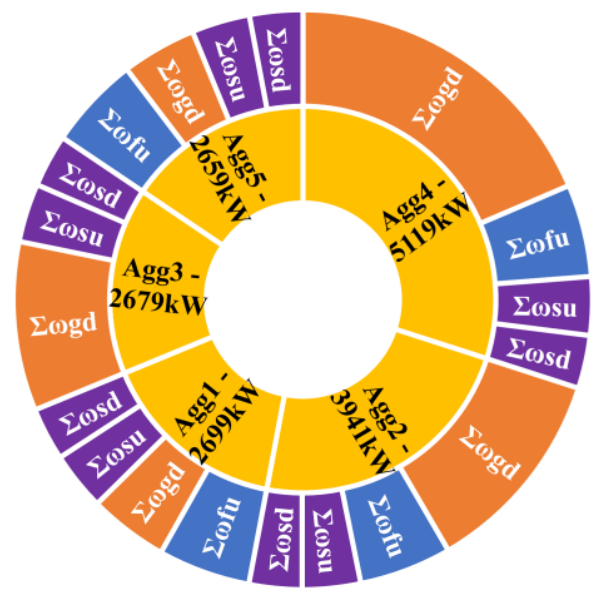

Figure 9. Distribution of the products traded in the market for each aggregator in the imbalance solving scenario. Blue, orange, and purple segments represent FLs, FGs, and BESSS, respectively.

By comparing the total quantity traded in both directions, it is possible to observe that equation (11) is satisfied. Note that FGs only trade downward products, but, as they are injecting power to the system, it can be assimilated to a FLs offering products in the upward direction. As a result, $\omega_{g, t}^{c, d}$ and $\omega_{g, t}^{e, d}$ are represented in the positive semi-axis of the figures.

A sensitivity analysis of the influence of the number of tangent lines $(n)$ is also performanced. Figure 10 assess on different error and time metrics of the linearized solution. Although all error metrics decrease with $n$, average, median and standard deviation parameters tend to stabilize when reaching 150 lines after $173.21 \mathrm{~s}$ of computation. 


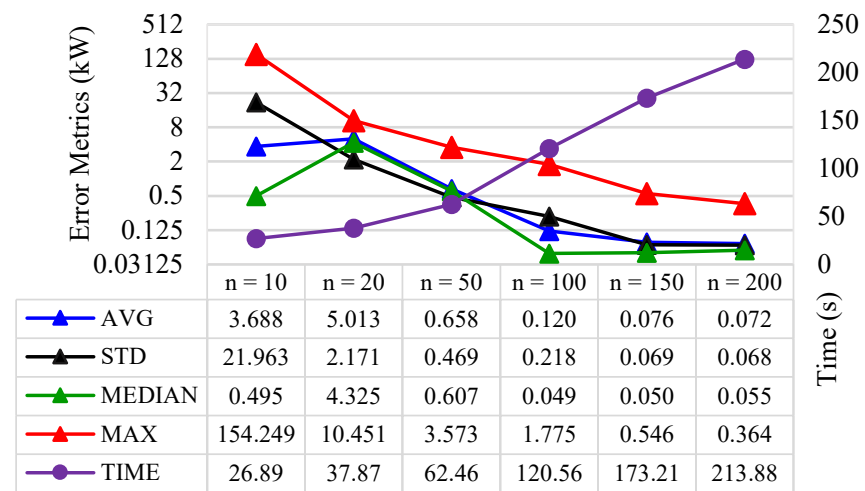

Figure 10. Metrics of the error $(\mathrm{kW})$ and computational time (s) considering different quantity of tangent lines ( $n$ ) for approximate non-linear restrictions in the imbalance scenario.

\section{Congestion Management}

The third scenario considers a congestion in the line 400-402. The associated power flow considering the baseload together with the profile after clearing the LFM is represented in Figure 11. It can be noted that the profile of the power flow thought line 400-402 after market clearing is slightly higher than baseload. This is due to the BESSs charging processes during the part of the day with no congestion issues to offer downward products when line is near its thermal limit.

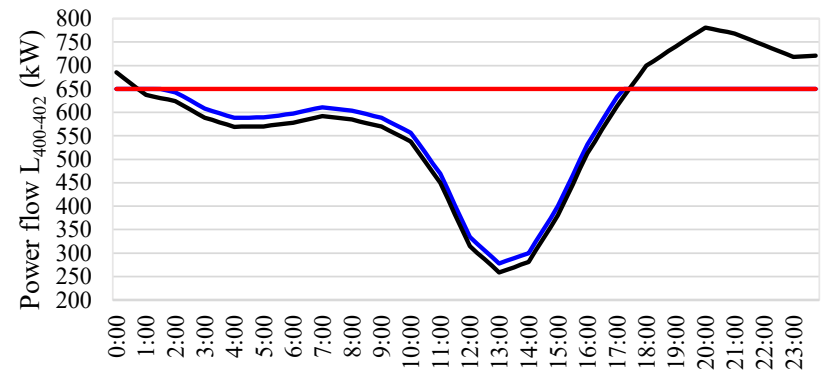

Figure 11. Aftermarket (blue), Forecasted (black) Power Flow through line 400-402 and its Thermal limit (red).

Figure 12 represents energy (lines) and capacity (bars) products traded to solve the congestion in the market. It can be noted that they follow the same profile that capacity products, as all pre-allocated capacity is consumed as energy $\left(\omega_{x, t}^{e}=\omega_{x, t}^{c} \cdot \Delta t\right)$.

The results minimize the total cost of acquiring flexibility in the LFM. As mentioned before, batteries of feeder downstream node 400 is charged between 1:00 and 17:00, while is discharged in the rest of the day. However, to maintain balance of the network, other Aggs offers downward products to globally equilibrate consumption. Only in this way, congestion is fully solved using local assets and products. As congestion is produced mainly at night-time hours, FGs cannot offer as much products as the other assets. Figure 13 represents a distribution of the hourly cost for congestion solving. Total costs amount to $103.85 €$ for a total of $1,871.2 \mathrm{kWh}$ of energy products and $7,484.8 \mathrm{~kW}$ of pre-allocated capacity products.

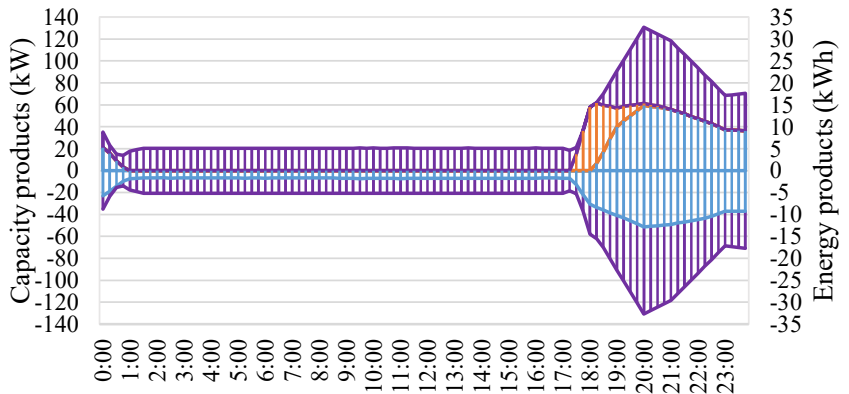

Figure 12. Energy (line) and capacity (bar) products traded in the congestion solving scenario. Blue, orange, and purple bars and line stands for FLs, FGs and BESSS, respectively. 


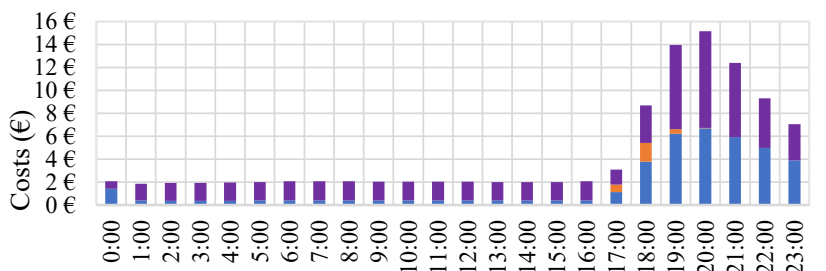

Figure 13. Hourly distribution of the costs for congestion scenario. Total FLs costs: 40.45€; Total FGs costs: 2.74€; Total BESSs costs: 60.66€. Quantity traded: 1,871.2 kWh.

Sensitivity study of the number of tangent lines $(n)$ affecting the overall precision of the linearization is also conducted for the congestion solving scenario. Figure 14 represents the metrics of the remaining imbalance presented after the LFM clearing as well as the computational time metric. Similarly to the imbalance scenario, median, average and standard deviation of the error stabilizes after $n=150$ with a total of $160.98 \mathrm{~s}$ of calculation time. As a result, no further computational effort is needed, due to the remaining imbalance maximum is $0.852 \mathrm{~kW}$ over $7,484.8 \mathrm{~kW}$.

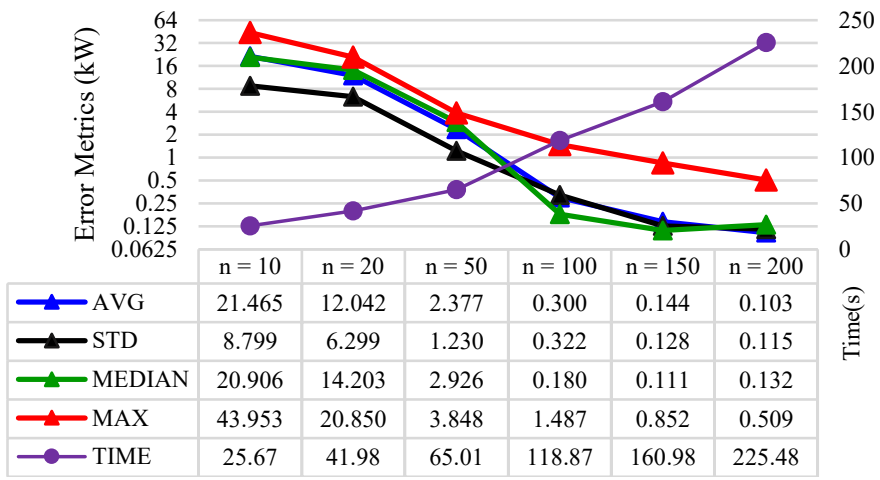

Figure 14. Metrics of the error $(\mathrm{kW})$ and computational time $(\mathrm{s})$ considering different quantity of tangent lines $(\mathrm{n})$ for approximate non-linear restrictions in the congestion scenario.

Agg 3 manages feeder where congestion takes place and, as a result, in Figure 15 can be noticed that half of the trading is carried out by that Agg, accumulating $3,828 \mathrm{~kW}$ of capacity products traded. Besides of that, it is perceived that congestion is mainly solved using BESSs products as previously mentioned, as most of the products traded by Agg 3 are from them. FLs also play an important role for solving congestion as its downward direction is highly traded in the market. FGs represent a near imperceptible amount, but it is indispensable to achieve economic efficiency.

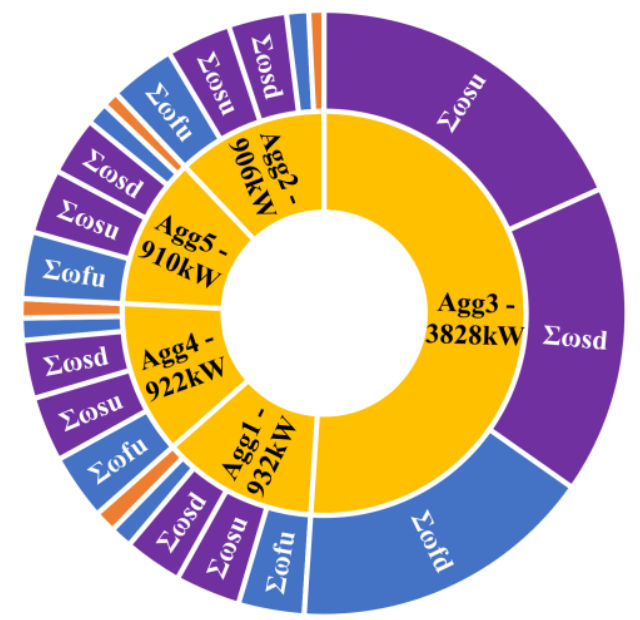

Figure 15. Distribution of capacity products traded in the market for each aggregator in the congestion scenario. Blue, orange, and purple segments represent FLs, FGs, and BESSs, respectively.

\section{CONCLUSION AND FUTURE LINES OF RESEARCH}

In this paper, a LFM for capacity and energy products trading is presented from the perspective of the LMO. The pre-allocation of the capacity products ensures market liquidity as it incentives assets to participate in the market. The proposed market formulation considers a linearization to ensure finding an approximation 
for global minimum of the objective function. Final LFM is achieved by transforming the initial NLP to a quadratic constrained problem and then, by means of using $n$ tangent lines, LP is obtained. LP ensures market efficiency as minimum quantity of product is traded for the two scenarios studied and global optimal finding. It has been also demonstrated that with a total of 150 lines is sufficient to obtain good performance as the maximum residual imbalance is less than $0.01 \%$ within a reasonable amount of time. Also, the charging-discharging behaviour of the BESSs is studied, as they are urged to maintain its initial SOC. That restriction affects power flowing through lines and products traded in the market.

Then, since the market can be seen from a P2P view, future lines of research will investigate the coordination of the agents in a P2P decentralized environment where no LMO is needed.

\section{ACKNOWLEDGEMENTS}

A. Paredes and J.A. Aguado acknowledge the support by FPU grant (FPU19/03791) founded by the Spanish Ministry of Education and Vocational Training (2020-2022), and the Spanish Ministry of Education Culture and Sports (2022-2024), also the support by the Universidad de Málaga to participate in scientific conferences.

\section{REFERENCES}

[1] IRENA, 'Renewable capacity statistics 2021 International Renewable Energy Agency (IRENA)', Abu Dhabi, 2021.

[2] OMIE, 'Evolution of the electricity market. Anual Report.', Madrid, Spain., 2020. [Online]. Available: https://bit.ly/3vDQq97.

[3] C. Ziras, C. Heinrich, and H. W. Bindner, 'Why baselines are not suited for local flexibility markets', Renewable and Sustainable Energy Reviews, vol. 135, no. December 2019, p. 110357, 2021, doi: 10.1016/j.rser.2020.110357.

[4] X. Jin, Q. Wu, and H. Jia, 'Local flexibility markets: Literature review on concepts, models and clearing methods', Applied Energy, vol. 261, p. 114387, Mar. 2020, doi: 10.1016/j.apenergy.2019.114387.

[5] The European Parliament and the Council of the European Union, Regulation (EU) 2019/943 of the European Parliament and of the Council of 5 June 2019 on the internal market for electricity, vol. 62, no. L158. 2019, pp. 54-191.

[6] T. Van Der Schoor and B. Scholtens, 'Power to the people: Local community initiatives and the transition to sustainable energy', Renewable and Sustainable Energy Reviews, vol. 43, pp. 666-675, 2015, doi: 10.1016/j.rser.2014.10.089.

[7] P. Olivella-Rosell et al., 'Optimization problem for meeting distribution system operator requests in local flexibility markets with distributed energy resources', Applied Energy, vol. 210, no. May 2017, pp. 881-895, 2018, doi: 10.1016/j.apenergy.2017.08.136.

[8] T. Schittekatte and L. Meeus, 'Flexibility markets: Q\&amp;A with project pioneers', Utilities Policy, vol. 63, p. 101017, Apr. 2020, doi: 10.1016/J.JUP.2020.101017.

[9] J. Villar, R. Bessa, and M. Matos, 'Flexibility products and markets: Literature review', Electric Power Systems Research, vol. 154, pp. 329-340, 2018, doi: 10.1016/j.epsr.2017.09.005.

[10] P. Olivella-Rosell et al., 'Local Flexibility Market Design for Aggregators Providing Multiple Flexibility Services at Distribution Network Level', Energies, vol. 11, no. 4, p. 822, Apr. 2018, doi: 10.3390/en11040822.

[11] A. Esmat, J. Usaola, and M. Á. Moreno, 'Distribution-level flexibility market for congestion management', Energies, vol. 11, no. 5, 2018, doi: 10.3390/en11051056.

[12] K. Zhang, S. Member, S. Troitzsch, S. Member, S. Hanif, and T. Hamacher, 'Coordinated Market Design for Peer-to-Peer Energy Trade and Ancillary Services in Distribution Grids', IEEE Transactions on Smart Grid, vol. 11, no. 4, pp. $2929-2941,2020$.

[13] F. Lezama, J. Soares, P. Hernandez-Leal, M. Kaisers, T. Pinto, and Z. Vale, 'Local Energy Markets: Paving the Path Toward Fully Transactive Energy Systems', IEEE Transactions on Power Systems, vol. 34, no. 5, pp. 4081-4088, 2019, doi: 10.1109/TPWRS.2018.2833959.

[14] OMIE, 'Working model of the Local Electricity Makets.', 2019. [Online]. Available: https://bit.ly/3b3DrpH

[15] J. Rosvall, S. Jansson, C. Klintberg, and A. Bengtsson, 'InterFlex Project - D8.12 Lessons learnt to draw business models in use case 4', 2019. [Online]. Available: https://bit.ly/3elaYO1.

[16] S. S. Torbaghan et al., 'A market-based framework for demand side flexibility scheduling and dispatching', Sustainable Energy, Grids and Networks, vol. 14, pp. 47-61, 2018, doi: 10.1016/j.segan.2018.03.003.

[17] C. Zhang, Y. Ding, N. C. Nordentoft, P. Pinson, and J. Østergaard, 'FLECH: A Danish market solution for DSO congestion management through DER flexibility services', Journal of Modern Power Systems and Clean Energy, vol. 2, no. 2, pp. 126-133, Jun. 2014, doi: 10.1007/s40565-014-0048-0.

[18] S. S. Torbaghan, N. Blaauwbroek, P. Nguyen, and M. Gibescu, 'Local market framework for exploiting flexibility from the end users', in 2016 13th International Conference on the European Energy Market (EEM), Jun. 2016, vol. 2016-July, pp. 1-6, doi: 10.1109/EEM.2016.7521304.

[19] D. Chattopadhyay and T. Alpcan, 'Capacity and Energy-Only Markets Under High Renewable Penetration', IEEE Transactions on Power Systems, vol. 31, no. 3, pp. 1692-1702, May 2016, doi: 10.1109/TPWRS.2015.2461675.

[20] OMIE, 'Day-ahead hourly price', 2021. [Online]. Available: https://www.omie.es/. 